\title{
Indocyanine green enhanced transpupillary thermotherapy of circumscribed choroidal haemangioma
}

\begin{abstract}
Purpose To investigate the effect of indocyanine green in enhancing the effect of diode laser for treatment of circumscribed choroidal haemangioma.

Methods Intravenous indocyanine green was used to enhance uptake of heat energy during transpupillary diode thermotherapy of circumscribed choroidal haemangioma. Results In a series of 6 patients treated in this manner, response to treatment was much improved compared with results without the use of indocyanine green. All patients retained the same or better visual acuity after treatment, and $67 \%$ of eyes improved visual acuity by more than 2 lines on Snellen testing. Ultrasonographic and angiographic evidence of improvement was seen in all patients. Treatment complications were minimal, comprising transient worsening of preexisting cystoid macular oedema in one case, and a small macular branch vein occlusion in the retina overlying the treated area.

Conclusions The use of indocyanine green as a contrast medium during transpupillary thermotherapy allows consistent uptake of diode laser energy, and shortens the duration of laser burn required. It is a cost-effective, easily performed outpatient procedure, with lower morbidity than other treatment modalities.
\end{abstract}

Key words Choroid, Haemangioma, Indocyanine green, Laser, Thermotherapy

Choroidal haemangiomas are uncommon intraocular tumours. These benign vascular hamartomas occur in two forms: a diffuse type and a circumscribed type. The diffuse type is commonly associated with Sturge-Weber syndrome. Circumscribed choroidal haemangioma is a distinct entity and differs from the diffuse type of haemangioma both clinically and histopathologically. ${ }^{1}$

Circumscribed choroidal haemangioma is a discrete, orange-red choroidal tumour commonly seen in the juxta-papillary or macular area and can result in poor visual acuity due to serous retinal detachment, cystoid macular oedema or a combination of both. ${ }^{2}$ Treatment options include cryotherapy, ${ }^{3}$ argon laser photocoagulation, ${ }^{4,5}$ external beam irradiation, ${ }^{6}$ brachytherapy ${ }^{7}$ or proton beam irradiation. ${ }^{8}$ More recently transpupillary thermotherapy using diode laser has been described in the management of circumscribed choroid haemangioma. $^{9-11}$

Transpupillary thermotherapy using infrared radiation $(805-801 \mathrm{~nm})$ from a diode laser delivers low-dose heat to the deeper layers of the retina and choroid, inducing tumour obliteration and resorption of sub-retinal fluid, and therefore may help to improve visual acuity., ${ }^{9,10}$ This particular mode of therapy has been used previously in the management of retinoblastoma and choroidal melanoma. ${ }^{12}$ More recently its use has been described in the treatment of circumscribed choroidal haemangioma. ${ }^{9-11}$

The use of intravenous indocyanine green with diode laser facilitates absorption of greater heat energy at the level of choroid and hence leads to more tumour destruction, which is probably due to occlusion and thrombosis of the vascular channels. Although this combination therapy has been described before in the management of recurrent amelanotic choroidal melanomas, ${ }^{12}$ to our knowledge this is the first time its use has been described for the treatment of circumscribed choroidal haemangioma. We describe our initial experience of 6 cases of circumscribed choroidal haemangioma treated with indocyanine green enhanced diode laser, managed between January 1998 and February 1999.

\section{Materials and methods}

An open prospective study was carried out between January 1998 and February 1999 at the Sheffield Ocular Oncology Service, Sheffield. Six patients with circumscribed choroidal
A. Kamal

A.R. Watts

I.G. Rennie

Academic Department of Ophthalmology

Royal Hallamshire Hospital Sheffield, UK

Professor I.G. Rennie Academic Department of Ophthalmology O Floor

Royal Hallamshire Hospital Glossop Road Sheffield S10 2JF, UK

Tel: $+(0) 1142669567$ Fax: $+(0) 1142766381$

Received: 14 February 2000 Accepted in revised form: 14 April 2000 
Table 1. Treatment details

\begin{tabular}{lll}
\hline Patient no. & Treatment details & Complications \\
\hline 1 & $12 \times 1200 \mathrm{~mW} \times 3 \mathrm{~mm}$ repeated at 4 weeks & Nil \\
2 & $14 \times 1100 \mathrm{~mW} \times 3 \mathrm{~mm}$ repeated at 10 weeks & Nil \\
3 & $10 \times 720 \mathrm{~mW} \times 3 \mathrm{~mm}$ & Nil \\
4 & $8 \times 1150 \mathrm{~mW} \times 3 \mathrm{~mm}$ & Transient exacerbation of CMO \\
5 & $12 \times 1000 \mathrm{~mW} \times 3 \mathrm{~mm}$ & Nil \\
6 & $11 \times 1250 \mathrm{~mW} \times 3 \mathrm{~mm}$ repeated at 15 weeks & BRVO overlying lesion \\
\hline
\end{tabular}

$\mathrm{CMO}$, cystoid macular oedema; $\mathrm{BRVO}$, branch retinal venous occlusion.

haemangioma received indocyanine green enhanced transpupillary thermotherapy using diode laser.

The diagnostic investigation included visual acuity assessment, slit-lamp biomicroscopy, fundoscopy, ocular ultrasonography, intravenous fluorescein angiography and intravenous indocyanine green angiography.

Treatment was performed as an outpatient procedure.

After obtaining written informed consent, retrobulbar anaesthesia with $2-3 \mathrm{ml}$ of $2 \%$ lignocaine was administered in all patients. Intravenous indocyanine green ( $25 \mathrm{mg}$ in $5 \mathrm{ml}$ of aqueous solvent) was injected intravenously $20 \mathrm{~s}$ prior to the treatment. Treatment was carried out using a slit-lamp-mounted Oculight SLx diode laser (Iris Medical) and a Volk Area Centralis Laser Plus or Volk Plus Super Quad 160 lens. Power used was between 720 and $1250 \mathrm{~mW}$ with a spot size of $3 \mathrm{~mm}$ and duration of $1 \mathrm{~min}$; the number of applications varied between 8 and 14 burns. Treatment was repeated once in 3 eyes as required (Table 1 ). The aim of the treatment was either obliteration of tumour or resolution of sub-retinal fluid, to treat tumour enlargement or visual impairment.

Patients were followed up at 4 weeks, 6 weeks and then on a 3-monthly basis with Snellen visual acuity, slitlamp biomicroscopy, colour fundus photography, fluorescein angiography and ocular ultrasound. Treatment-related complications were also recorded at each follow-up visit.

\section{Results}

Between January 1998 and February 1999 a total of 6 patients ( 4 male, 2 female) with circumscribed choroidal haemangioma were treated with intravenous indocyanine green enhanced transpupillary thermotherapy. Mean age at the time of treatment was 47 years (range 34-68 years).

Four patients experienced decreased or blurred vision prior to treatment, and 2 had photopsiae. Duration of symptoms ranged from 6 months to 4 years.
All tumours were solitary and unilateral. In 4 eyes, the tumour was located in the nasal quadrants $(67 \%)$ and in 2 eyes in temporal quadrants $(33 \%)$. Four tumours were juxta-papillary (67\%) and 2 were para-foveal (33\%).

Pre-treatment visual acuity ranged from counting fingers to 6/9 (Table 2). Clinical or fluorescein angiographic evidence of sub-retinal fluid was seen in all 6 eyes. Associated features observed were cystoid macular oedema in 2 eyes, and age-related macular degeneration in one eye.

Fluorescein angiography in all patients was consistent with the features of circumscribed choroidal haemangioma, demonstrating a characteristic early hyperfluorescence (Fig. 1b) and late leakage (Fig. 1c, d). Diagnostic intravenous indocyanine green angiography showed hyperfluorescence in early frames of less than $30 \mathrm{~s}$ (Fig. 1k), a mid-transit diffuse fluorescence at $5 \mathrm{~min}$ (Fig. 11) and a typical central washout of the dye in late frames at $30 \mathrm{~min}$ with persistent peripheral hyperfluorescence (Fig. $1 \mathrm{~m}$ ). Topographic series of indocyanine green angiography demonstrated the presence of sub-retinal fluid in all eyes.

The mean tumour thickness on ultrasonography was $2.5 \mathrm{~mm}$ (range 2.0-4.5 $\mathrm{mm}$ ); the mean tumour diameter was $7.75 \mathrm{~mm}$ (range $6.5-9 \mathrm{~mm}$ ) (Table 2). A-scan ultrasonography showed characteristic high internal reflectivity in all patients.

The main indication for treatment was decreased visual acuity in the affected eye or an increase in tumour size threatening the fovea. Intravenous indocyanine green enhanced transpupillary thermotherapy was used in all patients as described above, with treatment parameters as shown in Table 1.

Treatment was repeated in 3 eyes after a mean interval of 2 months (range 4-15 weeks). The mean parameters used in the first session were a power of $1070 \mathrm{~mW}$ (range $720-1250 \mathrm{~mW}$ ), with a mean of 11 applications for $1 \mathrm{~min}$ (range 8-12 applications) using a spot size of $3 \mathrm{~mm}$.

Table 2. Pre-and post-treatment visual acuity and ultrasound measurements

\begin{tabular}{|c|c|c|c|c|c|}
\hline \multirow[b]{2}{*}{ Patient no. } & \multirow{2}{*}{$\begin{array}{l}\text { Follow-up after } \\
\text { TTT (months) }\end{array}$} & \multicolumn{2}{|c|}{ Visual acuity } & \multicolumn{2}{|c|}{ Elevation $\times$ diameter } \\
\hline & & Before TTT & After TTT & Before TTT & After TTT \\
\hline 1 & 6 & $6 / 5$ & $6 / 5$ & $3 \times 8 \mathrm{~mm}$ & Flat \\
\hline 2 & 12 & $6 / 60$ & $6 / 18$ & $4.5 \times 8 \mathrm{~mm}$ & Flat \\
\hline 3 & 7 & $6 / 9$ & $6 / 5$ & $2 \times 6.5 \mathrm{~mm}$ & Flat \\
\hline 4 & 3 & $6 / 36$ & $6 / 36$ & $2 \times 7 \mathrm{~mm}$ & Flat \\
\hline 5 & 2 & $6 / 18$ & $6 / 9$ & $3 \times 9 \mathrm{~mm}$ & Flat \\
\hline 6 & 4 & CF & $4 / 60$ & $2 \times 8 \mathrm{~mm}$ & $1.2 \times 6.4 \mathrm{~mm}$ \\
\hline
\end{tabular}

TTT, transpupillary thermotherapy. 

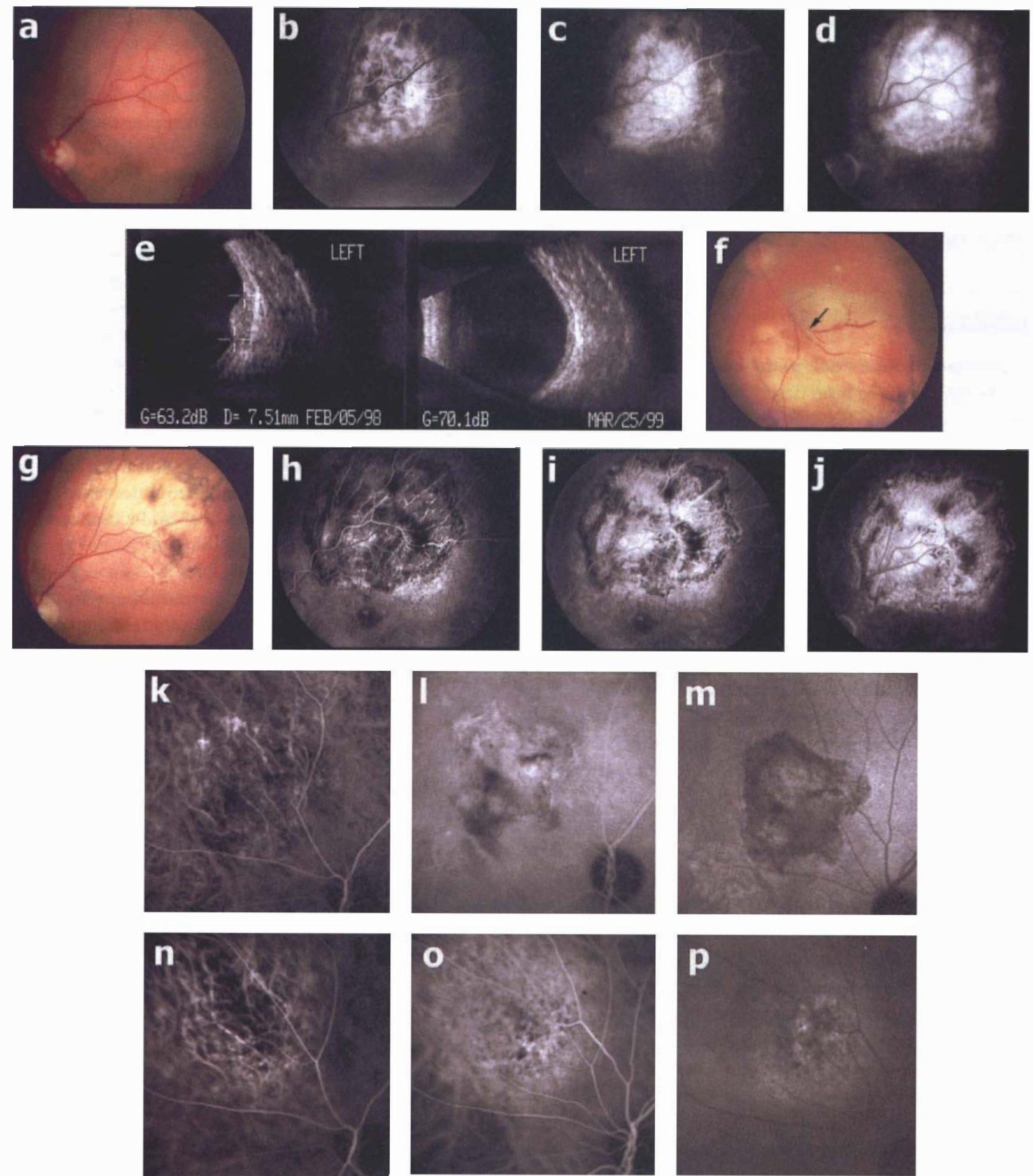

Fig. 1. (a) Colour photograph before treatment. (b) Early hyperfluorescence seen on the pre-treatment fluorescein angiogram. (c) Mid-transit of the pre-treatment fluorescein angiogram. (d) Late leakage seen on the pre-treatment fluorescein angiogram. (e) B-scan ultrasound scans before and after treatment. (f) Colour photograph of branch vein occlusion in treated area in one patient (arrow). (g) Colour photograph after treatment. ( $h$ ) Early phase of fluorescein angiogram after treatment. (i) Mid-phase of fluorescein angiogram after treatment. ( $j$ ) Late phase of fluorescein angiogram after treatment. (k) Early hyperfluorescence seen on indocyanine green angiogram before treatment. (l) Mid-transit diffuse fluorescence seen on indocyanine green angiogram before treatment. $(\mathrm{m})$ Late central washout seen on indocyanine green angiogram before treatment. $(n)$ Early phase of indocyanine green angiogram after treatment. (o) Mid-phase of indocyanine green angiogram after treatment. ( $p$ ) Late phase of indocyanine green angiogram after treatment.

Patients were followed up for a mean period of 6 months (range 3-12 months). Following the treatment all eyes showed tumour regression (Table 2).

At the last follow-up visit, tumour thickness was not measurable by ultrasound (Fig. 1e) in 5 eyes (83\%) and in 1 eye it had decreased from $2 \mathrm{~mm}$ to $1.2 \mathrm{~mm}$ (Table 2). Assessment of these eyes by fundoscopy showed a flat chorioretinal scar in 5 eyes (Fig. 1g) and, in 1 eye in which the tumour was close to the fovea, a mild persistent elevation. Post-treatment fluorescein angiography showed hypofluorescence, decrease or absence of leakage, and atrophic changes of the choroid (Fig. $1 \mathrm{~h}-\mathrm{j}$ ). Intravenous indocyanine green also confirmed a decrease in tumour vasculature and sub-retinal fluid (Fig. 1n-p). 
At the last follow-up visit, 4 eyes (67\%) showed an improvement in Snellen visual acuity by 2 or more lines, and in 2 eyes (33\%) vision remained stable (Table 2 ).

No complications were noted at the time of treatment. However, following the treatment 1 eye developed a small branch retinal vein occlusion (Fig. 1f), and another eye had a transient worsening of pre-existing cystoid macular oedema, which resolved with oral non-steroidal anti-inflammatory drugs.

\section{Discussion}

Several treatment modalities for circumscribed choroidal haemangioma have been described in the past, including argon laser, plaque brachytherapy, external beam radiation and proton beam irradiation. The most widely used conventional treatment is argon laser photocoagulation. ${ }^{2,4,13}$ Although limited success has been reported with this treatment, a study by Sanborn et al. ${ }^{4}$ raised the problems associated with argon laser photocoagulation, such as the need for repeated use of laser photocoagulation to decrease the sub-retinal fluid. Also the intense doses used in multiple sessions caused decreased visual acuity due to iatrogenic complications. Moreover, this study also reported a high tumour recurrence rate. In addition $70 \%$ of argon or krypton laser is absorbed by the retinal pigment epithelium, making it difficult to treat juxta-foveal lesions without incurring further damage to the fovea. ${ }^{14}$

A recent study by Schilling et al. ${ }^{6}$ described favourable results using low-dose lens-sparing external radiation. The therapeutic goal of this study was resorption of sub-retinal fluid rather than tumour destruction. A low dose of external irradiation of $20 \mathrm{~Gy}$ was used in 51 eyes. This study found that in about $39 \%$ of eyes visual acuity improved by more than 2 Snellen lines, in $22 \%$ it decreased whilst in $39 \%$ it remained stable. Sixty-four per cent of eyes in this series showed complete resolution of sub-retinal fluid over a period of 3-6 months, whilst $36 \%$ had residual sub-retinal fluid, and about $20 \%$ of cases required additional laser therapy. The functional success in this study was dependent on the lag duration between onset of symptoms and treatment. Radiation-induced side effects, such as cataract or retinopathy, were not reported.

Zografos et al. ${ }^{7}$ reported similar results using tumourdestructive cobalt- 60 brachytherapy in 41 symptomatic patients. Visual acuity was categorised on four different scales, and in two-thirds of cases an improvement by one or two categories was achieved over a period of 2 years. However, complete resolution of sub-retinal fluid with flat chorioretinal scar was noted in all cases. The overall outcome of this study was more or less comparable to that of the low-dose external radiation study, with about $15 \%$ of cases requiring complementary laser therapy. Complications including actinic maculopathy, retinal telangiectasia and diffuse retinal oedema were reported in this series. Although success was defined on clinical and visual outcome, none of these studies had pre- and post-treatment indocyanine angiography as described in our series.

Although both forms of radiation therapy produced favourable results with minimal complications, they each have their own disadvantages, such as irradiation of the whole eye with its related problems, the expense of the procedure, and the invasive nature of the surgery, with related surgical complications. Both techniques required hospital admission and $15-20 \%$ of cases required additional laser photocoagulation at a later date.

The beneficial effects of diode laser (near-infrared wavelength) have been reported in some earlier studies. ${ }^{14,15}$ These studies have shown that because of its greater penetration $(90 \%)$ of the optical media and minimal absorption (35\%) by the retinal pigment epithelium, diode laser spares most internal retinal structures such as the retinal vessels, nerve fibre layer and internal limiting membrane from damage. ${ }^{16}$ Therefore it may be assumed that diode laser can be less damaging than argon or krypton laser photocoagulation. ${ }^{17}$

The use of transpupillary thermotherapy in the management of circumscribed choroidal haemangioma has been reported recently. ${ }^{9-11}$ It acts by decreasing the size of the tumour and by stimulating resorption of sub-retinal fluid. Although the mechanism is not clear it is probable that the heat induced by the diode laser within the tumour leads to occlusion and thrombosis of the vascular channels. Occlusion of tumour vessels has been described in histopathological examination of choroidal melanoma treated with transpupillary thermotherapy. ${ }^{12,14}$

Few cases of circumscribed choroidal haemangioma treated with transpupillary thermotherapy have been reported in the literature. ${ }^{9,10}$ Nevertheless, in these cases, multiple sessions of therapy were required for effective treatment, and the duration of burn required was many times longer than in our study. This problem could be partly related to the minimal heat absorption by the vascular and non-pigmented circumscribed choroidal haemangioma. As intravenous indocyanine green is a protein-bound dye and remains in the choroidal circulation, it facilitates greater heat absorption at the level of choroid. ${ }^{18}$ Although its use has been described by Shields et al. ${ }^{12}$ for the treatment of amelanotic melanoma, this is the first report of indocyanine green enhanced thermotherapy of circumscribed choroidal haemangioma.

All patients in our series received intravenous indocyanine green $20 \mathrm{~s}$ prior to treatment, as described above. This allowed treatment to involve burns of $1 \mathrm{~min}$ duration, rather than the 3-6 min duration used without indocyanine green. ${ }^{11}$ Also, only $50 \%$ of patients required re-treatment, and these required only a single retreatment after a mean interval of 9 weeks. The tumour thickness in 2 of these eyes was greater than $4 \mathrm{~mm}$ and in 1 eye treatment was repeated as the tumour extended subfoveally, and hence was treated with minimal applications requiring two sessions. 
Post-treatment results were encouraging: $83 \%$ of eyes had complete regression of tumour ultrasonographically with a visible flat chorioretinal scar on ophthalmoscopy. This was evident on fluorescein and intravenous indocyanine green angiography, which showed decreased leakage, hypofluorescence and loss of tumour vasculature. However, one eye, with an extensive circumscribed choroidal haemangioma with sub-foveal extension, showed partial regression on ultrasound, due to limitation of treatment to maintain foveal function.

Visual acuity improved by more than 2 Snellen lines in $67 \%$ eyes, and remained stable in the remainder; none of the eyes dropped below their baseline visual acuity. These results are extremely encouraging when compared with the results of argon laser photocoagulation as reported in an earlier study.

Minimal and transient complications due to treatment were observed in only $33 \%$ of cases. In 1 eye there was a small branch macular vein occlusion over the surface of the tumour which did not cause any major visual problems (Fig. 1f). In another eye a transient worsening of cystoid macular oedema was observed which resolved with the use of oral non-steroidal anti-inflammatory drugs. Serious complications such as cellophane maculopathy, subretinal neovascularisation, persistent cystoid macular oedema and tumour recurrence, as described in earlier laser photocoagulation study, ${ }^{4}$ were not observed in this series.

In this pilot study, indocyanine green enhanced thermotherapy was effective in causing tumour regression and thereby improving visual acuity in most of the eyes. The advantages of this treatment include the shorter duration of burns required compared with transpupillary thermotherapy without indocyanine green enhancement, cost-effectiveness, the ease of performing the treatment as an outpatient procedure, the need for fewer sessions of therapy, and less damage to inner retinal structures with minimal treatment-related complications compared with argon laser photocoagulation. A limitation of transpupillary thermotherapy is its inability to treat peripherally situated tumours; however, as most circumscribed choroidal haemangiomas are situated in the posterior pole, and peripheral tumours are often asymptomatic, this factor may not be significant.

This study is non-randomised and reports a small group of patients with a rare tumour. However, in the short term, indocyanine green enhanced transpupillary thermotherapy appears to be an effective tool in the management of circumscribed choroidal haemangioma. Its longer-term effects will be evaluated in larger prospective trials.

\section{References}

1. Witschell H, Font LR. Haemangioma of choroid: a clinicopathologic study of 71 cases and review of literature. Surv Ophthalmol 1976;20:415-31.

2. Anand R, Augsburger JJ, Shields A. Circumscribed choroidal haemangiomas. Arch Ophthalmol 1989;107:1338-42.

3. Humphrey WT. Choroidal haemangioma response to cryotherapy. Ann Ophthalmol 1979;11:100-4.

4. Sanborn GE, Augsburger JJ, Shields JA. Treatment of circumscribed choroidal haemangiomas. Am J Ophthalmol 1982;89:1374-80.

5. Shields JA. The expanding role of laser photocoagulation for intraocular tumours: the 1993 Christian Zweng Memorial Lecture. Retina 1994;14:310-22.

6. Schilling H, Sauverin W, Lommatzsch A, et al. Long term results after low dose ocular irradiation for choroidal haemangiomas. Br J Ophthalmol 1997;81:267-73.

7. Zografos L, Bercher L, Chamot L, et al. Cobalt-60 treatment of choroidal haemangiomas. Am J Ophthalmol 1996;121:190-9.

8. Madreperla SA, Hungerford JL, Plowman PN, et al. Choroidal haemangioma: visual and anatomic results of treatment by photocoagulation or radiation therapy. Ophthalmology 1997;104:1773-9.

9. Lanzetta P, Virgili G, Ferrari E, Menchini U. Diode laser photocoagulation of choroidal haemangioma. Int Ophthalmol 1996;19:239-47.

10. Othmane IS, Shields CL, Shields JA, et al. Circumscribed choroidal haemangioma managed by transpupillary thermotherapy. Arch Ophthalmol 1999;117:136-7.

11. Garcia-Arumi J, Ramsay LS, Guraya BC. Transpupillary thermotherapy for circumscribed choroidal haemangiomas. Ophthalmology 2000;107:351-7.

12. Shields CL, Shields JA, Carter J, et al. Transpupillary thermotherapy for choroidal melanoma: tumour control and visual results in 100 consecutive cases. Ophthalmology 1998;105:581-90.

13. Augsberger JJ, Shields JA, Moffat KP. Circumscribed choroidal haemangiomas: long term visual prognosis. Retina 1981;1:56-61.

14. Brancato R, Pratesi R, Leoni G, et al. Histopathology of diode and argon laser lesions in rabbit retina: a comparative study. Invest Ophthalmol Vis Sci 1989;30:1504-10.

15. McHugh JDA, Marshall J, Capon M, et al. Transpupillary retinal photocoagulation in the eyes of rabbit and human using a diode laser. Laser Light Ophthalmol 1998;2:125-43.

16. Gabel VP, Birngruber R, Hillenkamp F. Visible and near infrared light absorption in pigment epithelium and choroid. In: Shimuzu K, editor. International congress series no. 450. XXI-II Concilium Ophthalmol Kyoto. Amsterdam, Oxford: Excerpta Medica, Elsevier, 1978:658-62.

17. Clover GM. The effects of argon and krypton photocoagulation on the retina: implications for the inner and outer blood retinal barriers. In: Gitter KA, Shatz H, Yannuzzi LA, et al., editors. Laser photocoagulation of retinal disease. San Francisco: Pacific Medical Press, 1998:11-8.

18. Chong LP, Ozler SA, de Queiroz JM Jr, Ligget PE. Indocyanine green-enhanced diode laser treatment of melanoma in a rabbit model. Retina 1993;13:251-9. 